\title{
An Energy-aware Hierarchical Architecture Design Scheme
}

\author{
Linfeng YUAN ${ }^{1}$, Yang XU ${ }^{1}, \mathrm{Xu} \mathrm{DU}^{2}$, Wenqing CHENG ${ }^{2}$ \\ ${ }^{1}$ Wuhan Maritime Communication Research Institute, Wuhan, P.R.China \\ ${ }^{2}$ Huazhong University of Science and Technology, Wuhan, P.R.China \\ E-mail: yuanlf@163.com
}

\begin{abstract}
Compared with the flat architecture in the design of sensor networks, the hierarchical architecture gains much attractive for the reason of scalability, management and energy efficiency. In order to distribute the energy evenly, nodes act the cluster head in some orders. The existing approaches don't pay a critical attention to the overhead during the role rotations. And the duration of a round is a priori, which is very application-specific. An energy-aware hierarchical architecture design scheme is put forward in this paper, namely, Adaptive Minimum Rotational Cost (AMRC) cluster formation scheme. The decision of beginning a new round is made adaptively by the cluster head itself. It combines the dynamic and static advantages in the clustering architecture. The simulation results demonstrate AMRC outperforms some other clustering protocols in many aspects.
\end{abstract}

Keywords: Sensor Network, Hierarchical Architecture, Energy-Aware, Role Rotation

\section{Introduction}

Sensor network technology, based on sensing, communication and computing research results, is a key technology for the future. Many future applications will rely on the embedded sensor network [1]. Sensor networks have spurred much interest in the networking research community recently.

Many questions must be dealt with in the design of the sensor network. Efficient routing protocol is one of those significant items. There are two main routing topologies in the sensor network: flat and hierarchical. The hierarchical (clustering) architecture gains much attractive for the reason of scalability, management and energy efficiency.

In the hierarchical architecture, each cluster includes one cluster head $(\mathrm{CH})$ and several common member nodes (MNs). MNs send the data to the $\mathrm{CH}$ during the communication and the $\mathrm{CH}$ send the gathered data to the sink node or to the other CHs. Usually, the MNs in one cluster will not communicate with each other directly, and the MNs in different clusters communicate with each other via the $\mathrm{CHs}$ in their clusters. So the $\mathrm{CHs}$ act the roles of "router" in the hierarchical architecture, they take more responsibility than the MNs and will consume more energy than the MNs.

Based on the role assigned to sensor nodes in a system, a hierarchical scheme can be grouped as a static one or a dynamic one [2]. In a static system [3][4], the $\mathrm{CHs}$ and their corresponding nodes will not change. So it is hard to minimize the system's energy consumption for non-optimized distances between a $\mathrm{CH}$ and its MNs or distribute energy cost among all the nodes. Dynamic mechanisms [5][6][7] can provide better fairness while remaining simplicity. In these dynamic schemes, clusters are periodically regenerated, and nodes take responsibility as the $\mathrm{CHs}$ in rotation. However, the regeneration of clusters is energy consuming. Therefore, it is desirable to design a hybrid scheme combining the advantages of both static and dynamic scheme [2].

In order to distribute the energy evenly, nodes act the $\mathrm{CH}$ in some orders. The existing approaches have solved the problems in many aspects [6][8][9][10], but they have two main disadvantages. First, they all don't pay a critical attention to the overhead during the role rotations. If the energy cost for the role rotations has added up to a certain degree, it will kill the benefits of hierarchical architecture. Second, these hierarchical protocols decide the beginning of a new round by some constant experimental periodic time. If the round time is too short, the rotational overhead will increase much. Or if the round time is long enough, when the $\mathrm{CH}$ has depleted its energy before the time of a new round, the system is "dead" in fact. Therefore, the selection of the duration time is closely dependent on the specific applications, 
which will greatly affect the scalability and management of the system.

In this paper, we put forward an adaptive minimum rotational cost (AMRC) energy efficient cluster formation scheme to minimize the rotational times and the rotational overhead. In AMRC, in order to minimize the rotational times, the node with the biggest residual energy will act the $\mathrm{CH}$ during the role rotations; in order to minimize the rotational overhead, one round will last for a period until the $\mathrm{CH}$ finds its residual energy has dropped to a certain threshold which is only decided by the CH's own energy. Therefore, the decision of beginning a new round is made adaptively by the $\mathrm{CH}$. It needs no information of the other nodes and needs no global information. Each cluster's rotation is independent and the rotation in one cluster will not affect the other clusters. So it combines both the dynamic advantages and static advantages in the hierarchical architecture. And, it is totally application-independent. It can not only distribute energy consumption in the network, but also reduce the overhead to prolong the system's lifetime.

The main contributions of this paper are as follows:

- Minimum rotational cost to reduce the overhead.

- Adaptive round time to meet any application's requirement.

- Combination of dynamic and static advantages in the hierarchical architecture.

The rest of the paper is organized as follows. Section 2 discusses some related work. Section 3 describes the AMRC scheme. Section 4 presents some experiments and compares AMRC with other schemes. The last section concludes this paper.

\section{Related Work}

In the flat routing architecture, each node typically plays the same role and sensor nodes collaborate to perform the sensing task. In hierarchical routing architecture, higher-level nodes $(\mathrm{CH})$ can be used to process and send the information, while low-level nodes $(\mathrm{MN})$ can be used to perform the sensing in the proximity of the target [11]. The hierarchical techniques can greatly contribute to overall system scalability, lifetime, and energy efficiency for load balancing and efficient resource utilization [2][5][6][7][11].

The $\mathrm{CH}$ will cost more energy than its MNs. In order to distribute energy consumption evenly among all the nodes including $\mathrm{CHs}$ and $\mathrm{MNs}$, role rotations will be used to let all nodes take turn to act the $\mathrm{CH}$ [2][5][6][7]. The selection of $\mathrm{CH}$ can be weight-associated $[4][12][13][14]$ or weight-independent [6][7]. The latter is simpler to implement and more robust in the presence of network dynamics while the former can produce more optimal clusters, which is more essential to the sensor networks. In weight-associated selection methods, the selection of $\mathrm{CHs}$ can be based on node ID [12][13], nodal degree [14], residual energy [4][6] or a combination of several parameters [5][12]. Since the energy consumption is more sensitive to the sensor network, the residual energy is often used as the criterion for $\mathrm{CH}$ selection. They all take the ratio, the node's residual energy to the sum of all nodes' residual energy, as the selection principle. Every node will need to know the sum either by a central controller or by computing on its own, it is hard or costly to get the computation results. In AMRC, each node is restricted within one cluster forever, so the selection of $\mathrm{CH}$ is very easy to be decided.

Many times of role rotations will take place in clustering formation, which will cost lots of energy too. But none of the existing cluster-based routing schemes have paid a critical attention to the overhead during the role rotations. Some have tried to reduce the rotation times by setting appropriate round period time. For example, LEACH [6] sets the round time to enable each node has enough energy to act $\mathrm{CH}$ once and act $\mathrm{MN}$ several times throughout the simulation lifetime. It gives no analysis about the number of role rotations and the energy consumption for these rotations. Though the network operation interval $\left(\mathrm{T}_{\mathrm{no}}\right)$ is forced to be much bigger than the clustering process interval $\left(\mathrm{T}_{\mathrm{cp}}\right)$ in HEED [5], the decision of its privilege, it will become the $\mathrm{CH}$ again if it has more energy than the other neighboring nodes. So this rotation is not necessary, and it will cost some energy among all the nodes in this cluster. In AMRC, each role rotation will happen in the same cluster and it will not affect any other clusters, different clusters have different number of role rotations and different duration time.

\section{AMRC Scheme}

\subsection{Connectivity Subnet}

It will efficiently decrease the overhead incurred by the interfaces among the clusters if each role rotation will only happen in its own cluster and it will not affect other clusters. So in AMRC, we restrict that the members in each cluster will not change throughout the whole transmission lifetime.

After the system runs in a steady state, the position of the sink node is relatively fixed. In order to make fully use of the position information in this protocol, the sink node lets all related nodes know its coordinates before the route discovery process. We need to establish a Connectivity Subnet (or Connectivity Set, CS). The CS is organized as follows. The sensing area is divided into sub-areas according to the position information, which must ensure the nodes in either sub-area can directly communicate with any node in the neighboring sub-area. 
GAF [15] has also put forward the concept of virtual grid to try to ensure the direct communication, but in fact, it cannot ensure the direct communication between the diagonal neighboring sub-area, as shown in Figure 1.

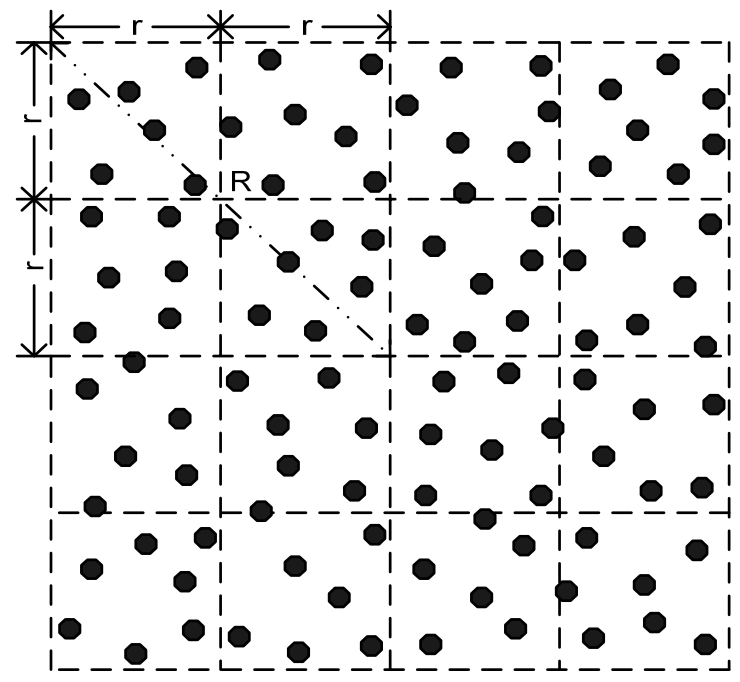

Figure 1. Connectivity subnet

We will establish the CS as follows. In Figure 1, the dashed line surrounds each sub-area and sixteen subareas are involved here. If the communication radius of each node is $\mathrm{R}$ and the square's length of each sub-area is $\mathrm{r}$, in order to ensure direct communication for any nodes in the neighboring sub-areas, $\mathrm{R}$ and $\mathrm{r}$ must meet the following requirement:

$$
(2 r)^{2}+(2 r)^{2} \leq R^{2}
$$

That is

$$
r \leq \sqrt{2} R / 4
$$

In this case, whichever node is selected as the $\mathrm{CH}$ in each cluster, it can directly communicate with the other $\mathrm{CHs}$ in the neighboring sub-areas. The restriction ensures that each role rotation can only take place in the same cluster, and it will not affect the other clusters. It also implies that different clusters can have different number of role rotations and different interval time.

\subsection{Decisive Rotational Threshold}

Since the role rotation in one cluster has no relationship with the other clusters, we first consider the rotation process within one cluster. Suppose the $i$ th node is the $\mathrm{CH}$ in a cluster at some time. If the $\mathrm{CH}$ finds its residual energy has decreased to some threshold, it will tell all the nodes in this cluster to begin a new round.

Denote $E_{i}(\mathrm{CH})$ as the energy when the node acted as the $\mathrm{CH}$ and $E_{i}(i)$ as its residual energy at present. In AMRC, the threshold to begin a new round is $\alpha \cdot E_{i}(C H)$, where $\alpha$ is a coefficient parameter of the threshold and it is determined before the system works. That is to say, if the energy of the $\mathrm{CH}$ has dropped to the $\alpha$ times of the energy when it acted the $\mathrm{CH}$ in this round, it needs to initiate a new round selection, as shown in the following term,

$$
E_{i}(i) \leq \alpha \cdot E_{i}(C H)
$$

Since the energy $E_{i}(\mathrm{CH})$ is different for each node and it is also different for each node in different round, the time to begin a new round is changeable. It means the role rotations will change adaptively.

\subsection{Cluster Formation}

There are two main issues in the design of the hierarchical architecture: the number of clusters and the cluster formation. We have discussed the first one in above subsection, now we consider the second issue.

In AMRC, because the nodes in a specific cluster will not change throughout the lifetime, the main problem in the cluster formation is to select a new $\mathrm{CH}$ within this cluster. If the old $\mathrm{CH}$ decides to begin a new round, it will broadcast an energy-request message (ReqEn) using a non-persistent carrier-sense multiple access (CSMA) MAC protocol. Each MN transmits its residual energy with an acknowledgement message $(A C K)$ back to the $\mathrm{CH}$. The $\mathrm{CH}$ compares all the MNs' residual energy and informs the node with the highest one to become the new $\mathrm{CH}$ in the coming round with an indication message $(I N D)$. The new $\mathrm{CH}$ sets up a TDMA schedule and transmits this schedule to the MNs in the cluster. After all nodes in the cluster know the TDMA schedule, the set-up phase is complete and the steady-state network operation phase (data transmission) can begin. Figure 2 gives the AMRC flowchart.

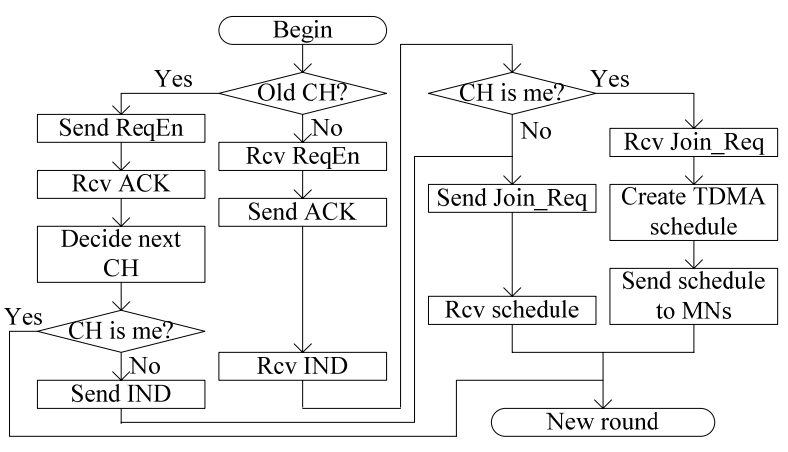

Figure 2. AMRC flow chart

\subsection{Some Discussions}

The design of hierarchical architecture can gain many benefits from the AMRC's solutions.

First, the process of AMRC ensures that the node with the highest residual energy will act the $\mathrm{CH}$ in the local area during the role rotations. Second, it is a prior for each node to know the parameters $k$ (number of clusters) and $N$ (total number of nodes) in LEACH-like protocols [5], while in AMRC, the decision to begin a 
new round is made adaptively by the $\mathrm{CH}$ own and it needs no information of the other nodes or any global information. Third, the working status is independent for each cluster and each node in one cluster will act the $\mathrm{CH}$ in some turns, so it combines the static and dynamic advantages successfully. Fourth, the duration of each round is determined adaptively, so it is not applicationspecific. The parameters $\alpha$ can be set with a bit smaller value when there are many packets should be transmitted after establishing the source-destination pair, so as to reduce the number of rotations. Or else, it can be set with a bit larger value when only a few packets need to deliver from the source node to the destination node, so as to balance the energy consumption more efficiently.

From these benefits, AMRC can reduce the number of rotations and minimize the rotational overhead significantly.

\section{Performance Simulation}

In this section, we evaluate the performance of AMRC protocol in the ns- 2 simulator. Some parameters are set as seen in Table 1.

Table 1. Parameters setting

\begin{tabular}{|l|l|l|}
\hline Parameter & Meaning & Value \\
\hline$M^{*} M$ & sensing region & $200 \mathrm{~m} * 200 \mathrm{~m}$ \\
$N$ & total number of nodes & 100 \\
$R$ & radio propagation range & $50 \mathrm{~m}$ \\
$E_{\text {elec }}$ & electronics energy & $50 \mathrm{~nJ} / \mathrm{bit}$ \\
$\varepsilon_{f s}$ & free space coefficient & $10 \mathrm{pJ} / \mathrm{bit} / \mathrm{m}^{2}$ \\
$\varepsilon_{m p}$ & multipath fading coefficient & $10 \mathrm{pJ} / \mathrm{bit} / \mathrm{m}^{2}$ \\
$L_{\text {data }}$ & data packet size & $100 \mathrm{bytes}$ \\
$E_{i}$ (max) & initial energy of each node & 1 Joules \\
\hline
\end{tabular}

We compare AMRC with LEACH-like protocols and evaluate the following performance metrics:

- The number of role rotations.

- Energy consumption in the transmission.

\subsection{The Number of Role Rotations}

We first examine the number of role rotations in LEACH-like protocols. In LEACH-like protocols, there are two steps in each round: the cluster process time $T_{c p}$ (set-up phase) and the network operation time $T_{n o}$ (steady-state phase). Along with the differences of the network operation time $T_{n o}$, the number of role rotations is also different. In our simulation, the network operation time $T_{n o}$ are set as 20,40,60,80 seconds, the number of role rotations is calculated every 100 seconds and the system lasts for 700 seconds. Figure 3 shows the simulation results.

From the figure, we can find that at the time of 700 second, the number of role rotations is 24 when Tno equals to 100 second, but it reaches 132 when Tno equals to 20 second. It indicates that Tno has a great influence to the number of role rotations in LEACH-like protocols.

While in AMRC protocol, the network operation time is not decided by manual operation and it is not application-specific, so it cannot influence the number of role rotations directly. It is the parameter of $\alpha$ that can decide the number of role rotations. With the same settings in LEACH-like protocol, we set $\alpha$ as $0.8,0.85$, $0.9,0.95$, and also examine the number of role rotations when the system works in the time from 100 second to 700 second. Figure 4 shows the simulation results.

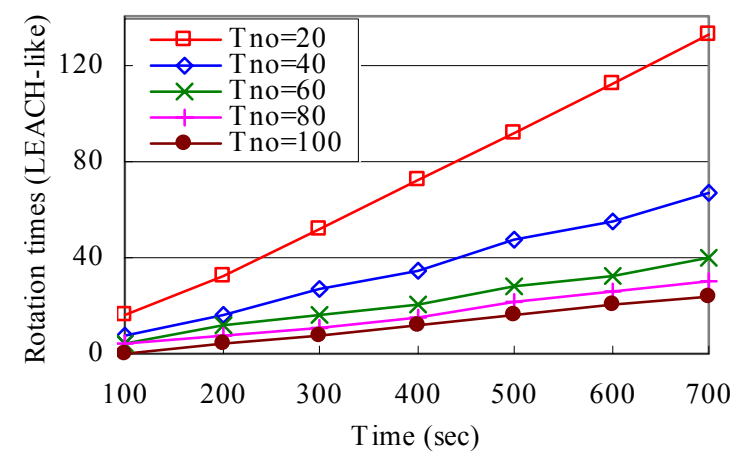

Figure 3. The number of role rotations in LEACH-like protocol

From Figure 4, when we compare the number of role rotations in the same times, we can find along with the increasing number of $\alpha$, the number of role rotations gets larger. At the time of 700 second, the number of role rotations is 4 when $\alpha$ equals to 0.8 , and it is 22 when $\alpha$ equals to 0.95 . This implies that the decisive rotation threshold is more likely satisfied when $\alpha$ is smaller.

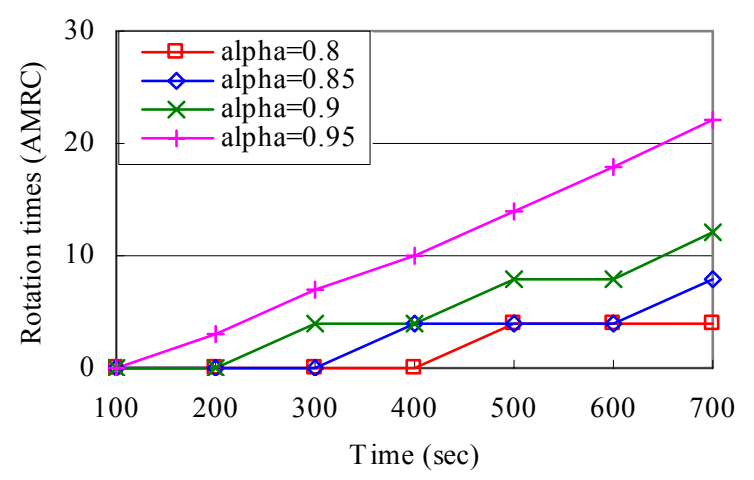

Figure 4. The number of role rotations in AMRC protocol

Comparing Figure 3 and Figure 4, we can also find that at the same time, all the number of role rotations in AMRC protocol is smaller than those in LEACH-like protocols, even that of the worst curve in Figure 4 ( $\alpha=0.95$ ) is smaller than that of the best curve in Figure $3\left(T_{n o}=100\right)$. In fact, $\alpha$ equals to 0.95 means that the $\mathrm{CH}$ will begin a new round when it detects that its 
current energy has dropped $5 \%$, but $5 \%$ is not necessary for the $\mathrm{CH}$ to give up its $\mathrm{CH}$ 's privilege, and that $\mathrm{CH}$ can continue to do more jobs at that time.

\subsection{Energy Consumption in the Transmission}

We set the network operation time as $20,40,60,80$ seconds in LEACH-like protocol and set $\alpha$ as 0.9 in AMRC protocol, Figure 5 shows the energy consumption of all the nodes during the transmission per 100 seconds.

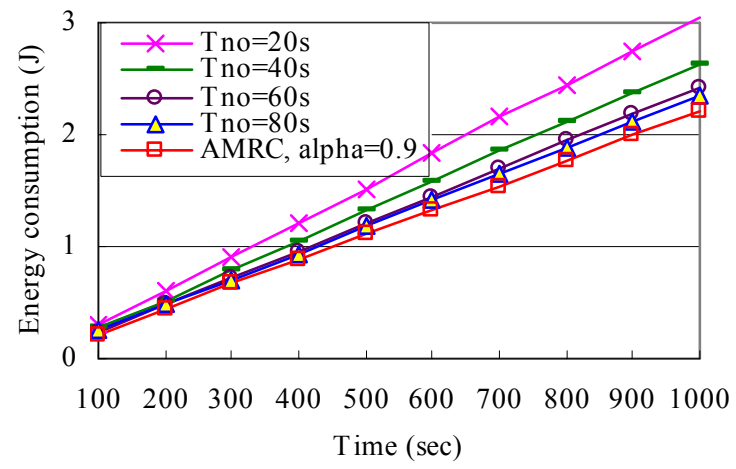

Figure 5. Energy consumption

In LEACH-like protocols, the energy consumption gets smaller when the network operation time is longer. The differences of energy consumption come from the different number of the role rotations. This implies that from the view of the energy efficiency, the role rotations cannot be neglected in the hierarchical architecture system. In AMRC protocol with $\alpha$ equals to 0.9 , the energy consumption is smaller than those in LEACHlike protocols at each corresponding time. It is for the reason that decreasing the number of role rotations that the energy consumption is reduced too. It can be expected that when $\alpha$ gets smaller, the energy consumption for all nodes will reduce further.

\section{Conclusion}

The LEACH-like hierarchical protocols suffered from the overhead for the role rotations and they are application-specific. In this paper, the AMRC energy efficient scheme is put forward to minimize the number of role rotations and the rotational overhead. Each cluster can take its own rotation without affecting the other clusters. And the adaptive round time makes it widely used in any application scenarios.

\section{Acknowledgement}

This work is supported by the National Natural Science Foundation of China (No.60572049) and the Natural Science Foundation of Hubei Province, P.R.China (No. 2005ABA264).

\section{References}

[1] John A. Stankovic, Tarek F. Abdelzaher, Chenyang Lu, Lui Sha, Jennifer C. Hou, "Real-Time Communication and Coordination in Embedded Sensor Networks", Proceedings of the IEEE, July 2003, Vol.91, No.7, pp.1002-1022.

[2] Quanhong Wang, Hossam Hassanein, Glen Takahara. "Stochastic Modeling of Distributed, Dynamic, Randomized Clustering Protocols for Wireless Sensor Networks", Proceedings of the 2004 International Conference on Parallel Processing Workshops, pp.1-8.

[3] C. F. Chiasserini, I. Chlamtac, P. Monti, and A. Nucci, "Energy Efficient Design of Wireless Ad Hoc Networks", Proc. of Networking 2002, Lecture Notes in Computer Science (LNCS), Italy, May 2002, No.2345, pp.387-398.

[4] S. Ghiasi, a. Srivastava, X. Yang, and M. Sarrafzadeh, "Optimal Energy Aware Clustering in Sensor Networks", Sensors Magazine, 2002, Vol.19, No.2, pp.258-269.

[5] O. Younis, and S. Fahmy. "Distributed Clustering in Adhoc Sensor Networks: A Hybrid, Energy-Efficient Approach", IEEE INFOCOM 2004, March 2004, Vol.1, pp.629-640.

[6] W.B.Heinzelman, A.P.Chandrakasan, H.Balakrishnan. "An Applicationspecific Protocol Architecture for Wireless Microsensor Networks", IEEE Transactions on Wireless Communications, Oct. 2002, Vol.1, No.4, pp.660-670.

[7] S. Bandyopadhyay and E. J. Coyle, "An energy efficient hierarchical clustering algorithm for Wireless Sensor Networks", IEEE INFOCOM 2003, Vol.3, pp.1713-1723.

[8] Mark Perillo and Wendi Heinzelman, "DAPR: A Protocol for Wireless Sensor Networks Utilizing an Application-based Routing Cost", IEEE WCNC 2004, Vol.3, March 2004, pp.1540-1545.

[9] Yan Yu, Ramesh Govindan, Deborah Estrin. "Geographical and Energy Aware Routing: a recursive data dissemination protocol for wireless sensor networks", In Seventh Annual ACM/IEEE International Conference on Mobile Computing and Networking (MobiCom'00), August 2000.

[10] Fan Ye, Haiyun Luo, Jerry Cheng, et al. "A Two-tier Data Dissemination Model for Large-scale Wireless Sensor Networks". In Proc. of the 8th Annual International Conf. on Mobile Computing and Networking (MobiCom'02), September 2002, pp.148159.

[11] Jamal N. Al-karaki, Ahmed E. Kamal, "Routing techniques in wireless sensor networks: a survey", Wireless Communications, IEEE [see also IEEE Personal Communications], vol: 11, issue: 6, Dec 2004, pp. 6-28.

[12] D. J. Baker, and A. Ephremides, "The architecture organization of a mobile radio network via a distributed algorithm", IEEE Tran. On Communications (Legacy, pre 1988), vol.29, issue: 11, Nov. 1981, pp.1694-1701.

[13] A. Ephremides, J.E.Wieselthier, and D.J. Baker, "A 
design concept for reliable mobile radio networks with frequency hopping signaling", Proceedings of IEEE, vol.75, Issue: 1, Jan. 1987, pp. 56-73.

[14] M. Gerla, and J. T. C. Tsai, "Multi cluster, mobile, multimedia radio network", Wireless Networks, vol.1, issue: 3,1995 , pp.255-265.
[15] Xu Y, Heidemann J, and Estrin D. "Geography-informed Energy Conservation for Ad Hoc Routing". In Proc. 7th Annual International Conference on Mobile Computing and Networking (MobiCOM 2001). July 2001, pp. 70-84. 